\title{
Aberration-Corrected Transmission Electron Microscopy Reveals Nanoscale Disorder in Bismuth Ferrite Single Crystals
}

\author{
K. Urban ${ }^{1}$, C. L. Jia ${ }^{1,2}$, L. Jin ${ }^{1}$, S. B. Mi ${ }^{2}$, M. Alexe ${ }^{3}$, D. Hesse ${ }^{4}$ \\ ${ }^{1}$ Peter Grünberg Institute \& Ernst Ruska Center, Research Center Jülich, D-52425 Jülich, Germany. \\ 2 International Center for Dielectric Research, Xi'an Jiaotong University, Xi'an 710049, China. \\ ${ }^{3}$ Department of Physics, University of Warwick, Coventry CV4 7AL, United Kingdom. \\ ${ }^{4}$ Max Planck Institute of Microstructure Physics, Weinberg 2, D-06120 Halle, Germany.
}

Bismuth ferrite $\left(\mathrm{BiFeO}_{3}, \mathrm{BFO}\right)$ is an intrinsic multiferroic that simultaneously shows antiferromagnetic and ferroelectric properties at room temperature [1]. This makes it a candidate material for devices functioning on the basis of an electric control of magnetic order.

In recent years, in particular after it was demonstrated [2] that thin epitactic films of BFO display extraordinary high values of spontaneous electric polarization, the work on electric and magnetic properties and their coupling concentrated on thin film systems. Also the structure of polarization-domain walls in these films was studied by transmission electron microscopy [e.g. 3-6]. In our work we followed an entirely different approach. It is generally accepted that for a study of the basic, intrinsic physical properties of a material single crystals are required. In fact, compared to thin films there is comparatively little work done on BFO single crystals. And in spite of more than thirty years of BFO research, until recently [7] there was not a single study on single crystals by electron microscopy. Here we give a brief account of the first results obtained on single crystals by aberration-corrected TEM [8]. Our study is typical for the high potential of aberration-corrected high-resolution TEM in the field of modern materials science where structures of atomic- to nanoscale may be decisive for physical properties but are not always noticed by non-local techniques.

The growth of $\mathrm{BFO}$ single crystals is difficult since, on account of the volatility of $\mathrm{Bi}_{2} \mathrm{O}_{3}$ and the instability of $\mathrm{BFO}$ at high temperatures, thermodynamic and kinetic aspects play a role. We have grown $\mathrm{BFO}$ single crystals employing the established flux-growth technique from a $\mathrm{Bi}_{2} \mathrm{O}_{3} / \mathrm{Fe}_{2} \mathrm{O}_{3} / \mathrm{B}_{2} \mathrm{O}_{3}$ flux, at a mol ratio of 4/1/0.8 [9]. The resulting [001]-oriented crystals had a diameter of some millimeters and a thickness of about $300 \mu \mathrm{m}$. From these samples for TEM with foil normals parallel to two orthogonal directions, $[-1,-1,0]$ and $[1,-1,0]$ were cut by FEI Helios FIB milling followed by a finish by means of a Fischione 1040 NanoMill. Electron microscopy was carried out at $300 \mathrm{keV}$ in a FEI Titan 80-300 TEM equipped with an image spherical-aberration corrector. The NCSI technique $[10,11]$ was used throughout. In contrast to conventional imaging modes it gives strong (bright on a dark background) contrast for all atoms, including oxygen. To see oxygen is a prerequisite for measuring both the magnetic moments and the electric polarization vector, unit cell by unit cell. Prior to electron microscopy the single crystals were characterized by piezoresponse force microscopy in order to demonstrate ferroelectric hysteresis and measure the Curie temperature and by SQUID magnetometry to demonstrate the antiferromagnetic ordering and to measure the appertaining Neél temperature. This proved that the single crystals studied show the established set of physical properties [1] and are typical for state-of-the-art BFO single crystals.

Figure 1(a) shows the first important feature characterizing the BFO single crystals. Between a (horizontal) electric polarization stripe domain boundary pattern we find a contiguous vertex domain pattern filling the entire crystal. This pattern is the projection of a tetrahedral arrangement of nanodomain walls. 
On the basis of an atomic resolution study these walls could be identified as $180^{\circ}$ walls. These nanodomains give rise to satellites at $\{1 / 2,1 / 2,1 / 2\}$ type reflections in electron diffraction patterns corresponding to a characteristic length of $15.5 \mathrm{~nm}$. This challenges the established interpretation of the $\{1 / 2,1 / 2,1 / 2\}$ type satellites observed in neutron scattering experiments in terms of a long-range cy-cloidal spin ordering.
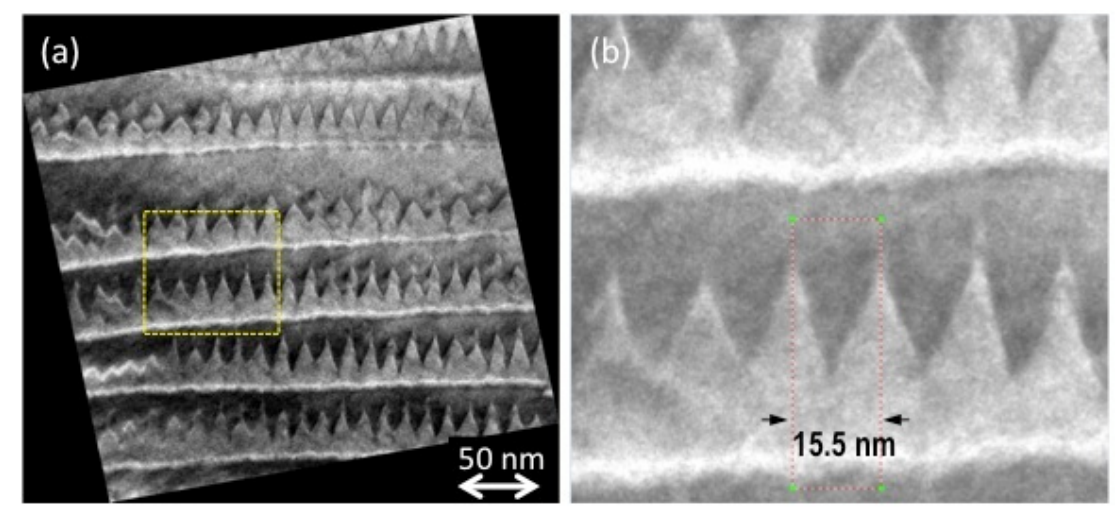

Figure 1. (a) The contiguous vertex domain pattern inside the stripe domains. (b) Magnification of the framed area in (a). The vertex distance of $15.5 \mathrm{~nm}$ corresponds to the location of the satellite spots at $\{1 / 2,1 / 2,1 / 2\}$ positions.

The second characteristic feature revealed by our atomic-resolution TEM study is the existence of atomic- to nanoscale disorder in form of random oxygen-octahedron tilts out of the ideal crystallographic [111] directions typical for the rhombohedral structure with R3c space group. According to a recent theory the octahedron tilts can be correlated with magnetic (dis)order [12]. Since this disorder does not allow to form the long-range magnetic cycloid also this observation challenges the generally accepted view that the weak ferromagnetism observed in BFO single crystals is related to the cycloidal spin ordering. We note that the domain structure and atom- to nanoscale disorder observed in our study is not only much too small in scale to be detectable by the low-resolution characterization tools so far applied to BFO single crystals, also the disorder averages out to zero over larger areas and thus could not be detected by the more integral techniques applied in BFO research before.

\section{References:}

[1] G. Catalan and J. F. Scott, Adv. Mater. 21 (2009) p. 2463.

[2] J. Wang et al. Science 299 (2003) p. 1719.

[3] J. Seidel et al. Nature Mater. 8 (2009) p. 229.

[4] C. T. Nelson et al. Nano Lett. 11 (2011) p. 828.

[5] A. Lubk et al. Nano Letters 13 (2013) p. 1410.

[6] Y. Wang et al. Phys. Rev. Lett. 110 (2013) p. 267601.

[7] A. Berger et al. Phys. Rev. B 85 (2012) p. 064104-1.

[8] C. L. Jia et al., submitted for publication.

[9] F. Kubel and H. Schmid, J. Cryst. Growth 129 (1993) p. 515.

[10] C. L. Jia, M. Lentzen and K. Urban, Science 299 (2003) p. 870.

[11] C. L. Jia et al. Microsc. Microanal. 19 (2013) p. 310.

[12] L. Bellaiche, Z. Gui and A. I. Kornev, J. Phys.: Cond. Matter 24 (2012) p. 312201-1. 